\title{
MÉTHODE DE MESURE RADIOGRAPHIQUE DE L'ÉPAISSEUR DES TISSUS ADIPEUX SOUS-CUTANÉS, CHEZ LE PORC.
}

PAR

\section{B. L. DUMONT et R. FÉVRIER}

Station de Recherches sur l'Elevage C. N. R. Z., Jouy-en-Josas.

\section{INTRODUCTION}

Le corps du porc est entouré par un tissu adipeux qui représente, selon MCMEEKan (I), plus de $20 \mathrm{p}$. IOO du poids vif et plus des $2 / 3$ des tissus gras totaux d'un porc de roo $\mathrm{kg}$.

Ce tissu adipeux sous-cutané est particulièrement important dans les parties supérieures des régions dorsales et lombaires.

A ce niveau, il est prélevé sur la carcasse, dans la découpe parisienne, sous forme d'un morceau appelé bardière ou lard dorsal (2).

L'importance de ce morceau et, par suite, la valeur commerciale de 1a carcasse, sont étroitement liées à son épaisseur $(3-4-5-6)$.

D'autre part, au cours de la croissance du porc, les tissus adipeux présentent l'allométrie la plus marquée (7). Les variations quantitatives du tissu adipeux permettent donc, en première analyse, de connaitre l'évolution de la composition corporelle pendant la croissance.

La mesure de l'épaisseur du tissu adipeux sous-cutané constitue un moyen de connaitre ces variations et, par conséquent, d'étudier quelques aspects de la croissance du porc.

L'utilisation de la radiographie permet d'obtenir cette mesure sur l'animal vivant, avec beaucoup de précision. Elle évite de recourir à la méthode des abattages successifs qui devient lourde et coûteuse dès qu'elle porte sur de gros animaux.

(1) Ce travail a été réalisé avec la collaboration de MM. Jean RE'TtaGLlati et Jean Weber. 


\section{I. - PRINCIPES D' UTILISATION DE LA RADIOGRAPHIE POUR L'EVALUATION DES DÉPOTS GRAISSEUX DE L'ORGANISME}

\section{Généralités.}

Les trois principaux tissus du corps possèdent des transparences différentes aux rayons $X$. Le tissu osseux est le plus opaque ; le tissu adipeux, au contraire, est le moins opaque. L'opacité du tissu musculaire et celle du tissu adipeux, bien que voisines, permettent cependant de préci-

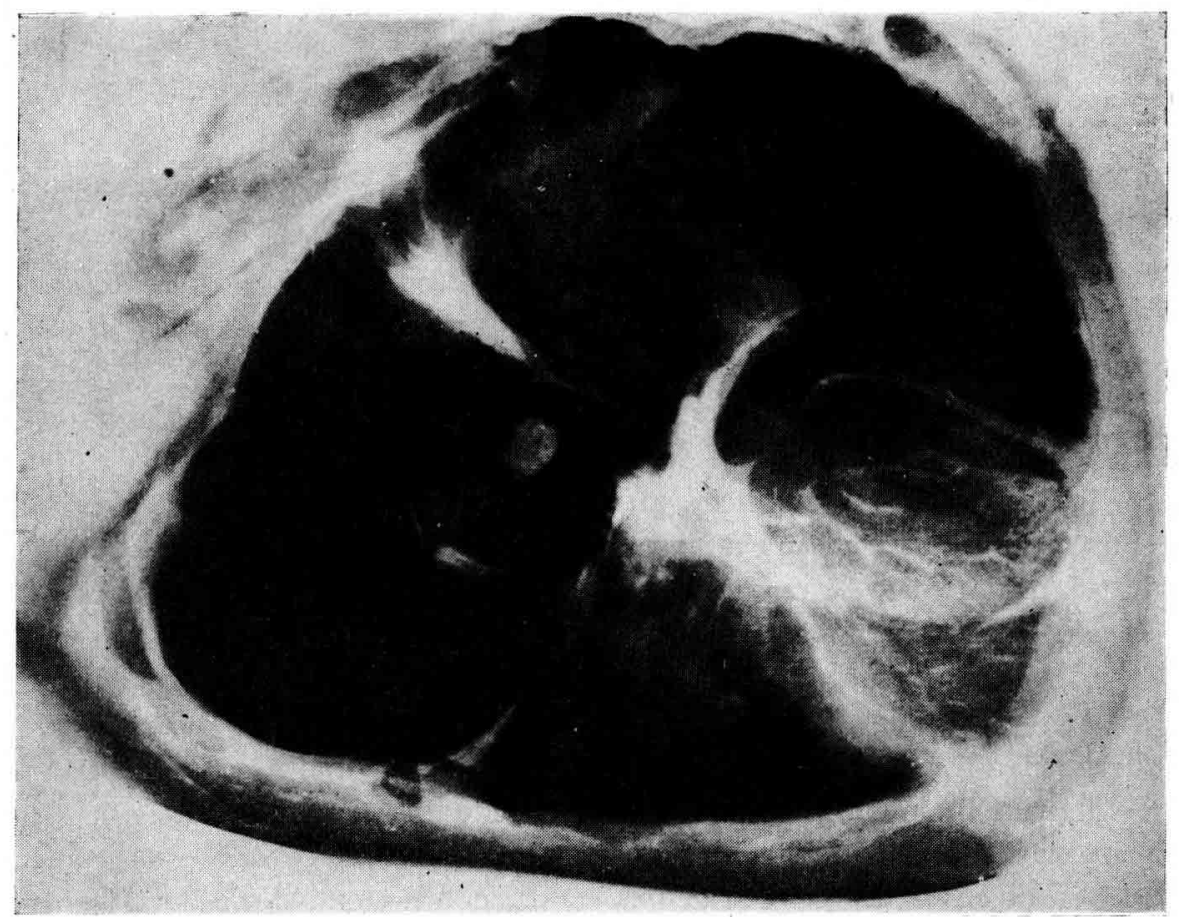

FIG I. - Radiographie d'une tranche de viande de 2 cm d'épaisseur prélevée dans la partie moyenne du membre postérieur perpendiculairement au fémur. On distingue de l'extérieur vers le centre:

-.- la couche de gras sous-cutané qui entoure le membre postérieur,

- les muscles cruraux,

- le fémur qui apparaît en noir très fonçé, au centre du cliché.

ser les limites de séparation de ces deux tissus, lorsqu'ils sont examinés sous la même épaisseur (fig. I).

Dans les régions superficielles du corps, lorsque ces deux tissus se présentent en couches superposées (le tissu adipeux étant le plus externe), l'examen radiographique, sous des incidences de profil, permet de les distinguer et d'apprécier leur épaisseur relative. 
La mesure de l'épaisseur du tissu adipeux sous-cutané par cette technique a été introduite par NyLIN, chez l'homme, en rg29 (8).

Depuis, elle a permis, par des prises de clichés en différents endroits du corps, d'apprécier d'adiposité des sujets et d'étudier certaines de ses principales causes de variation. L'ensemble des travaux sur ce sujet a été récemment passé en revue par KEYs et BROzEK (9).

\section{Application au porc.}

La couche de lard est limitée, dans le plan médian du corps :

- supérieurement, par la ligne médiane du dos,

- inférieurement, par une ligne sensiblement tangente aux bords

FIG 2. -- Radiographie de la couche de lard. On distingue successivement :

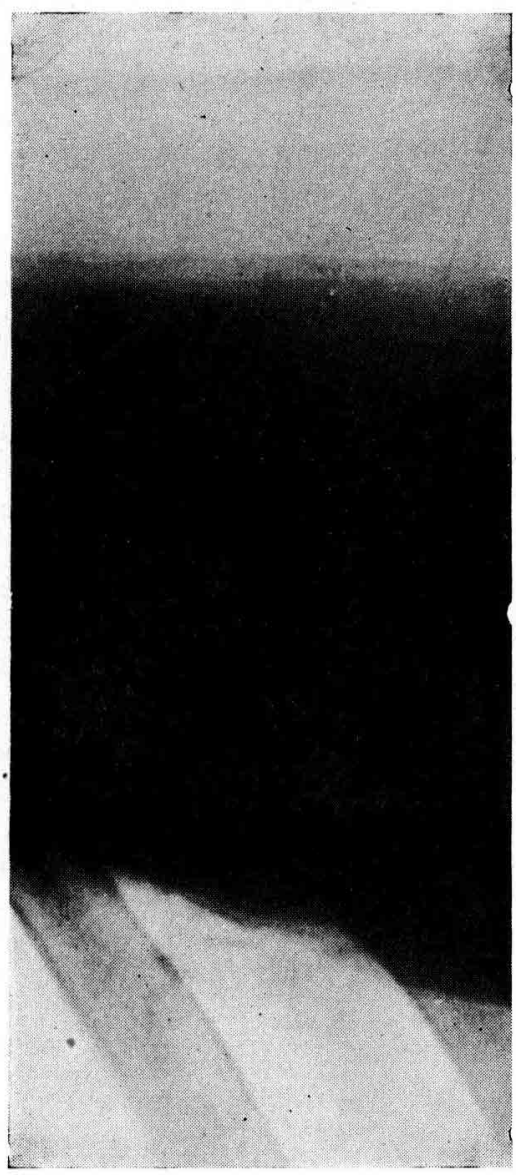

« le milieu extérieur

$\leftarrow$ la peau

$\leftarrow$ la couche externe du lard

$\prec$ - limite des 2 couches

$\leftarrow$ la couche interne du lard

$\leftarrow$ limite inférieure de la couche de lard

$\leftarrow$ zone des apophyses épineuses des vertèbres (au centre de la photo on voit se dessiner le contour de l'une d'elles).

supérieurs des apophyses épineuses des vertèbres dorsales et lombaires.

Elle est donc comprise, à ce niveau, entre deux milieux d'absorption très différents : 1'air et l'os. 
Elle apparait, par suite, sur le cliché radiographique, comme une plage de densité moyenne, encadrée par une plage externe très noire et une plage interne nettement plus claire où se dessine l'ombre portée des apophyses épineuses des vertèbres.

L'épaisseur de cette plage de densité intermédiaire représente l'épaisseur de la couche de lard (fig. 2).

Différents auteurs ont déjà utilisé la radiographie pour mesurer l'épaisseur de lard chez le porc (IO-II-I2), sans toutefois préciser les conditions pratiques d'utilisation ; certains points importants, notamment l'agrandissement de l'image de la couche de lard, semblent leur avoir échappé.

Le travail que nous rapportons ici résume l'expérience pratique que nous avons de cette méthode, introduite par nous dans notre Station en I 952.

\section{II. - TECHNIQUE.}

\section{I. - Matériel utilisé.}

\section{a) Appareillage :}

Les résultats que nous rapportons ici ont été obtenus avec un appareillage simple : appareil portatif, dont les principales caractéristiques sont les suivantes :

- caractéristiques électriques ;

Det1x tensions possibles pour le tube : $72 \mathrm{KV}$ et ro $\mathrm{mA}$ (" forte pénétration ») et $57 \mathrm{KV}$ et io $\mathrm{mA}$ (" faible pénétration »).

- finesse de l'image:

dimensions utiles du foyer : $\mathrm{I}, \mathrm{O} \times \mathrm{I}, \mathrm{O} \mathrm{mm}$.

- filtre permanent.

Les rayons, en quittant le foyer, doivent traverser la fenêtre de verre $\mathrm{du}$ tube à rayons $\mathrm{X}$, une couche d'huile et le capot de la fenêtre en philite. I. 'ensemble équivaut à un filtre de moins de $0,5 \mathrm{~mm}$ d'aluminium.

- minuterie électrique :

réglable par dixième de seconde, entre o et Io secondes.

Comme le laissent prévoir ces caractéristiques, les possibilités d'emploi de l'appareil sont assez limitées. I1 offre, par contre, 1'avantage d'être aisément transportable et, dans la gamme des appareillages de radiographie, d'être relativement peu onéreux.

\section{b) Matériel divers.}

Film Kodak Regulix,

Ecrans renforçateurs super-rapides,

Filtre $0,5 \mathrm{~mm}$ d'aluminium,

Cassette porte-film, épaisseur I2 mm. 


\section{II. - Mode opératoire.}

\section{a) Formation de l'image :}

Un faisceau de rayons $\mathrm{X}$ (fig. 3) est centré sur la région à examiner (AB) et impressionne un film radiographique placé dans une cassette portefilm qui est parallèle au plan médian du porc ( $\left.A^{\prime} B^{\prime} C^{\prime} D^{\prime}\right)$.

L'image de la couche de lard se forme sur le film en A'B', entre l'extrémité supérieure des apophyses épineuses et la ligne supérieure du dos.

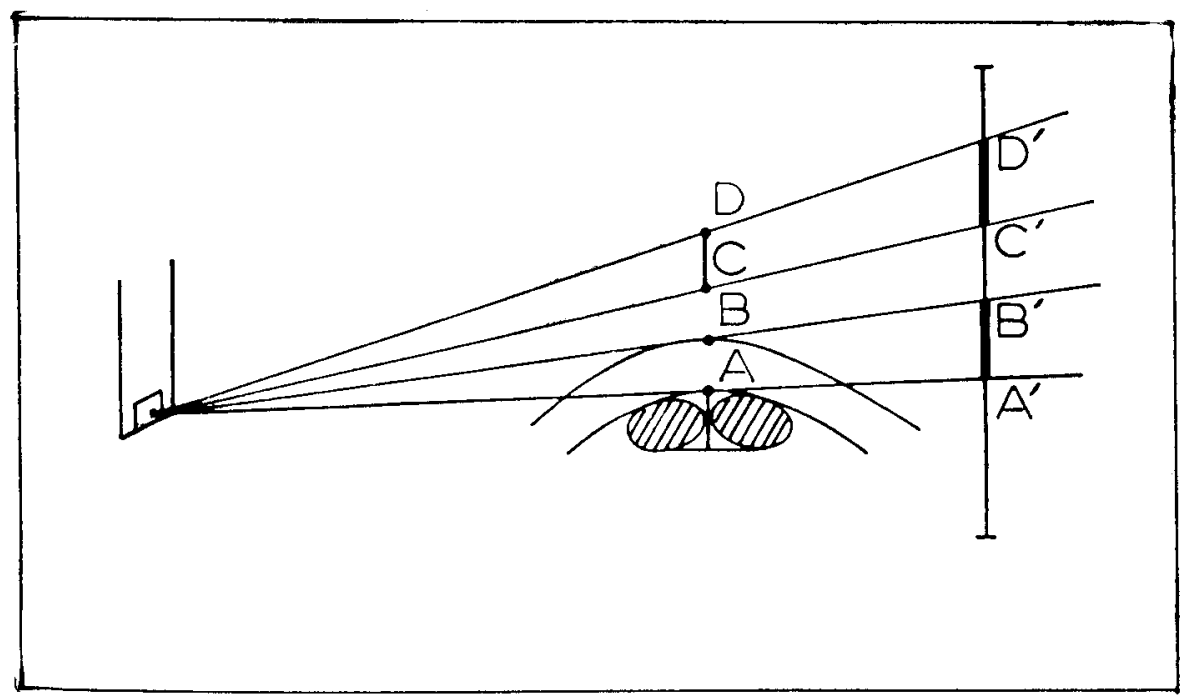

FIG. 3. - Formation de l'image radiographique.

Un témoin métallique, de longueur connue (CD), placé dans le plan médian du corps, permet de calculer l'agrandissement subi par les images radiographiques des objets placés dans le plan médian.

$x$ : représente l'épaisseur réelle de la couche de lard,

$l:$ est la longueur du témoin métallique,

$x^{\prime}$ : représente l'épaisseur de l'image de la couche de lard,

$l$ ': est la longueur de l'image du témoin métallique.

On a :

$$
\frac{x}{l}=\frac{x^{\prime}}{l^{\prime}}
$$

d'où :

$$
x=\frac{x^{\prime} \cdot l}{l^{\prime}} \text {. }
$$

\section{b) Position de l'animal :}

Les prises de clichés peuvent se faire sur animal debout ou sur animal couché sur le côté.

Il est très important de surveiller, dans les deux cas, le parallélisme 
entre le plan médian du porc et la cassette porte-film, et de placer le repère exactement dans le plan médian. Pour cela, quand l'animal est couché, nous plaçons le témoin métallique sur une planchette à laquelle est fixé un niveau à bulle.

La correspondance des mesures faites dans les deux positions est très bonne.

\section{c) Temps de pose.}

Les temps de pose utilisés sont consignés dans le tableau I.

Dans les intervalles de poids marqués, ils s'appliquent à des porcs d'épaisseur moyenne. Il y aurait lieu de les majorer légèrement pour des porcs plus épais que la normale.

TABLEAU I

Temps de pose en secoudes.

\begin{tabular}{|c|c|c|c|}
\hline \multirow{2}{*}{ Poids du porc en $\mathrm{kg}$} & \multicolumn{3}{|c|}{ Lieux de prise du cliché } \\
\hline & Cou & Dos & Rein \\
\hline 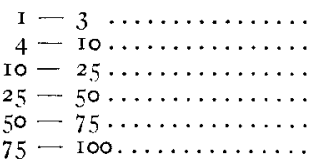 & $\begin{array}{c}0,2 \\
0,3 \\
0,4 \\
0,5 \\
0,6 \\
0,7-0,8\end{array}$ & $\begin{array}{c}0,2 \\
0,3 \\
0,3 \\
0,4 \\
0,5 \\
0,6-0,7\end{array}$ & $\begin{array}{c}0,2 \\
0,2 \\
0,3 \\
0,4 \\
0,5 \\
0,6--0,7\end{array}$ \\
\hline
\end{tabular}

Note. - Ces temps de pose s'entendent pour:

- un film Kodak Regulix,

- une distance foyer film de $70 \mathrm{~cm}$,

- un appareil muni d'un filtre de $0,5 \mathrm{~mm}$ d'aluminium.

\section{III. - RÉSULTATS ET DISCUSSION.}

Après avoir mis au point ces techniques d'obtention d'une image radiographique nette, nous avons voulu éprouver la validité des résultats qu'elle permet d'obtenir en les comparant à ceux que fournit, au même niveau, la lecture sur la carcasse, après fente de l'animal suivant son plan médian par une coupe sagittale de la colonne vertébrale.

I a mesure faite sur la carcasse peut être prise comme mesure de référence. Il convient toutefois de la considérer avec une certaine prudence.

Tout d'abord, pour procéder à la section du corps dans le sens 1ongitudinal, celui-ci doit être suspendu et l'effet de traction qu'il subit est susceptible de modifier la disposition de la couche de lard et, de la sorte, son épaisseur. 
De plus, il peut arriver que cette section ne soit pas rigoureusement effectuée dans le plan médian, et la lecture de l'épaisseur du lard s'en trouve affectée.

Pour estimer la précision avec laquelle la mesure de l'épaisseur du lard peut être effectuée, nous avons déterminé cette dernière sur les deux moitiés du même animal. I es résultats obtenus ont été les suivants (tableau II).

TABLEAU II

\begin{tabular}{|c|c|c|c|}
\hline Niveau de la mesure $\ldots \ldots \ldots \ldots$. & Cou (1) & $\operatorname{Dos}\left({ }^{2}\right)$ & $\operatorname{Rein}\left({ }^{3}\right)$ \\
\hline $\begin{array}{l}\text { Nombre de couples de mesures...... } \\
\text { Epaisseur moyenne du côté gauche de }\end{array}$ & 57 & 57 & 57 \\
\hline $\begin{array}{l}\text { la carcasse } \ldots \ldots \ldots \ldots \ldots \ldots \ldots \ldots \bar{X}_{1} \\
\text { Epaisseur moyenne du côté droit de }\end{array}$ & $43,15 \mathrm{~mm}$ & $29,28 \mathrm{~mm}$ & $35 \mathrm{~mm}$ \\
\hline $\begin{array}{l}\text { la carcasse..................... } \\
\text { Différence gauche - droite }\end{array}$ & $42,93 \mathrm{~mm}$ & $29, \mathrm{I} \quad \mathrm{mm}$ & $34,87 \mathrm{~mm}$ \\
\hline 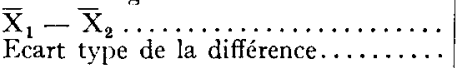 & $\begin{array}{l}+0,22 * \mathrm{~mm} \\
+\mathbf{I}, 35 \mathrm{~mm}\end{array}$ & $\begin{array}{l}+0,18^{*} \mathrm{~mm} \\
\pm 0,88 \mathrm{~mm}\end{array}$ & $\begin{array}{l}+0,13^{*} \mathrm{~mm} \\
\pm 1,17 \mathrm{~mm}\end{array}$ \\
\hline \multicolumn{4}{|c|}{$\begin{array}{l}\left({ }^{1}\right) \text { au niveau de la première vertèbre dorsale } \\
\left({ }^{2}\right) \text { au niveau de la demière côte } \\
\left({ }^{3}\right) \text { au niveau de la dernière vertèbre lombaire } \\
(*) \text { non signifiquatif }\end{array}$} \\
\hline
\end{tabular}

I a comparaison des mesures prises sur la carcasse et sur le cliché radiographique nous a donné les résultats suivants (tableau III).

TABLEAU III

\begin{tabular}{|c|c|c|c|}
\hline Niveau de la mesure & Cou & Dos & Rein \\
\hline Nombre de mesures.... & 60 & 60 & 60 \\
\hline Moyenne carcasse $\ldots \ldots \ldots \ldots \ldots \ldots \bar{x}_{1}$ & $46,35 \mathrm{~mm}$ & $28,50 \mathrm{~mm}$ & $3^{2,60} \mathrm{~mm}$ \\
\hline 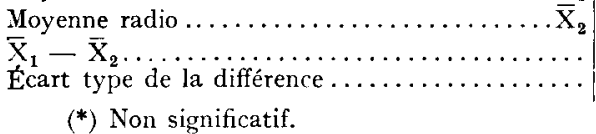 & $\begin{array}{r}47,44 \mathrm{~mm} \\
-1,09^{*} \mathrm{~mm} \\
\pm \quad 1,17 \mathrm{~mm}\end{array}$ & $\begin{array}{r}30,55 \mathrm{~mm} \\
-2,05^{*} \mathrm{~mm} \\
+0,90 \mathrm{~mm}\end{array}$ & $\begin{array}{r}30,59 \mathrm{~mm} \\
+\quad 2,0 \mathrm{I}^{*} \mathrm{~mm} \\
+\quad \mathrm{I}, \mathrm{I} 2 \mathrm{~mm}\end{array}$ \\
\hline
\end{tabular}

Par conséquent, les erreurs que peuvent dơnner les images du cliché radiographique par rapport à la lecture directe sur la carcasse, présentent une distribution voisine de celle que donnent les lectures d'une moitié de la carcasse par rapport à l'autre moitié. Compte tenu de la précision demandée et de l'objectif poursuivi, la précision obtenue paraît acceptable.

\section{Causes d'erreurs. - Précautions à prendre.}

I,es causes de discordances entre la mesure sur la carcasse et la mesure sur le cliché, en dehors de celles qui concernent la lecture sur la carcasse, sont les suivantes :

Mauvais contrôle du parallélisme entre le rapport d'agrandissement et la cassette porte-film. 
Mauvaise position du témoin par rapport au plan médian du porc. Mauvaise position de l'animal.

Erreurs de lecture sur la radio :

a) de l'épaisseur de la couche de lard,

b) de la longueur du témoin.

Il est indispensable de faire apparaître les apophyses épineuses des vertèbres pour fixer avec précision la limite inférieure de la couche de lard.

FIG 4. - Cliché sous-exposé. Il présente 3 zones:

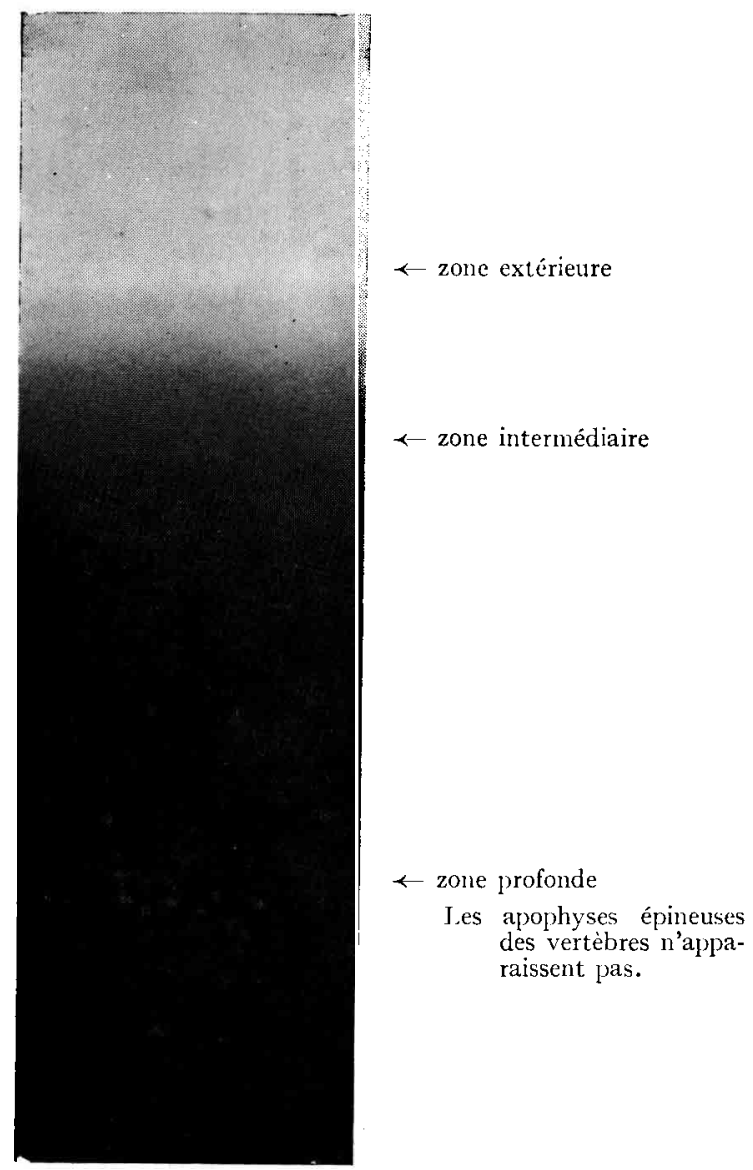

Il arrive que, pour des porcs plus épais que la moyenne, les temps de pose indiqués dans le tableau I ne suffisent pas. Il est difficile, pour des gros porcs, de dépasser le temps d'exposition indiqué, en raison du flou possible résultant de la respiration. Dans ce cas, les conditions de prise (I seconde, $72 \mathrm{KV}$, Io $\mathrm{mA}$ ) ne permettent pas d'obtenir sur le cliché 
les tissus osseux. Ia radio présente alors trois zones caractéristiques : une zone extérieure très noire,

une zone blanche,

une zone intermédiaire grisâtre (fig. 4).

L'épaisseur de cette zone intermédiaire ne représente pas nécessairement, comme on pourrait le croire, l'épaisseur du lard. Ia figure 5 indique l'importance de l'erreur commise aux différents endroits, entre les mesures prises sur le cliché et celles prises sur la carcasse, chez des porcs de IOO $\mathrm{kg}$.

Ces différences s'expliquent de la façon suivante: les conditions théoriques de la formation de 1'image représentée dans la figure 3 ne sont pratiquement jamais réalisées, et notamment le bord supérieur de l'apophyse épineuse des vertèbres est presque toujours en-dessous du niveau supérieur des masses musculaires latérales. La conséquence de cette différence de niveau est que la partie supérieure des masses musculaires projette sur le cliché, au-dessus des apophyses épineuses, une ombre portée (fig. 6) qui se superpose à 1'ombre propre du lard et, par suite, en masque le bord inférieur. Les différences enregistrées dans ce cas dépendent, d'une part de la différence du niveau entre les masses musculaires et les apophyses épineuses, et d'autre part du centrage du flux de rayons $X$.

Ce rebondi des masses musculaires par rapport à la colonne vertébrale est particulièrement important dans la région postérieure du rein et celle du sacrum où était prise la troisième mesure " rein ". Il explique les différences importantes entre les mesures radio et les mesures carcasse, et il interdit la prise de mesure radiographique à ce niveau avec ce genre d'appareil pour des porcs d'un poids supérieur à roo $\mathrm{kg}$. A ce niveau, en effet, la couche de lard ne repose plus sur ces apophyses épineuses des vertèbres, mais sur des masses musculaires.

La qualité gétnérale des clichés obtenus est assez mauvaise, manquant nettement de contraste. Ce fait résulte des possibilités très limitées de l'appareil en intensité (Io $\mathrm{mA}$ ), qui obligent à utiliser, pour compenser cette insuffisance, des films très rapides et desécrans renforçateurs ultrarapides.

L'utilisation d'un appareillage plus puissant (appareil fixe à 2 ou 4 kenotrons) permettrait sans doute d'augmenter suffisamment le contraste des clichés et, par suite, de permettre des mesures précises au niveau du sacrum. L'augmentation de puissance autoriserait des prises de clichés à grande distance foyer-film, ce qui supprimerait, en rendant les rayons $\mathrm{X}$ pratiquement parallèles, les rapports d'agrandissement, et supprimerait par là les causes d'erreurs possibles introduites par son utilisation. 

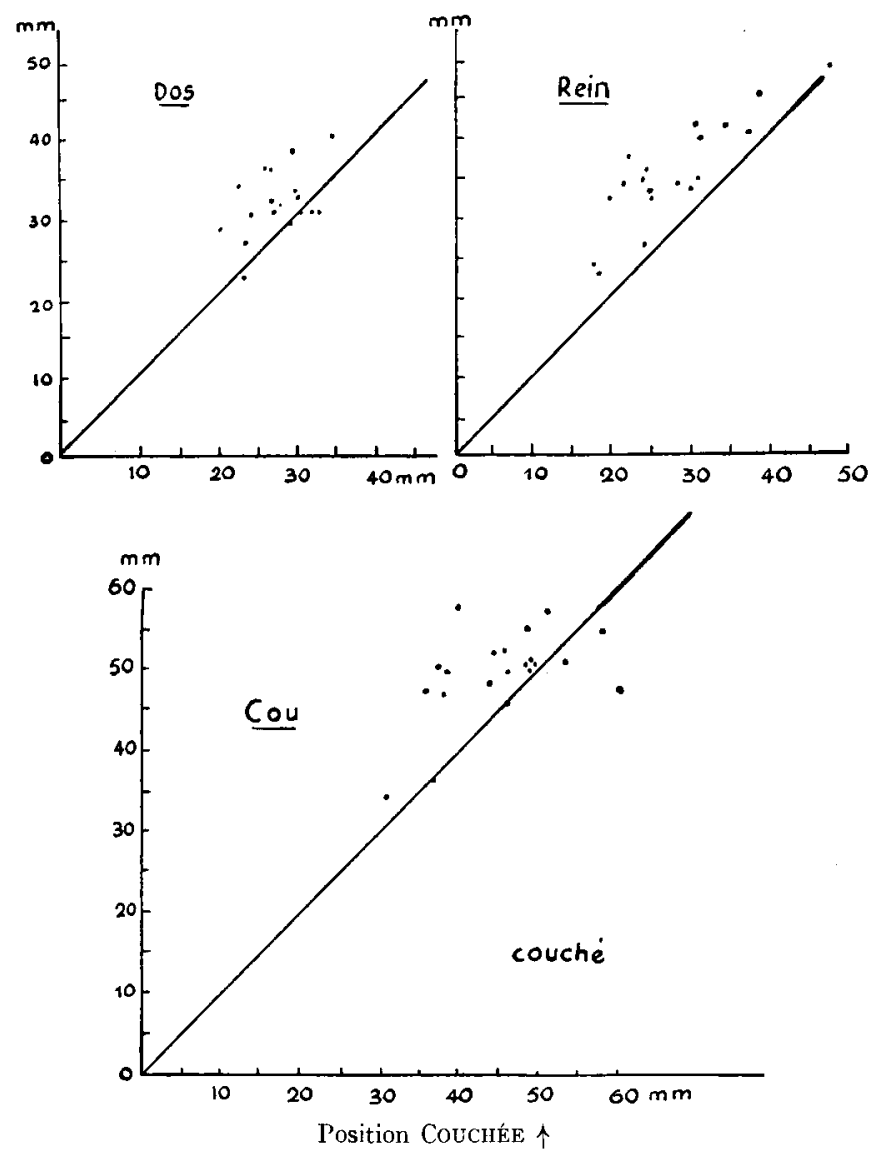

Position DEBOUT $\downarrow$
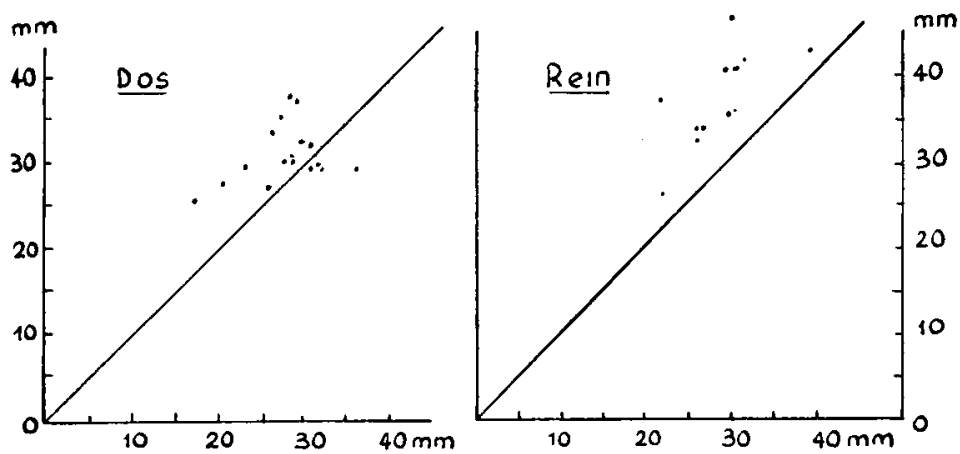

Fig 5. - Comparaison des résultats obtenus par lectures de clichés sous-exposés et les résultats fournis par la carcasse.

En abscisses : lectures de l'épaisseur de lard sur clichés sous-exposés.

En ordonnée : épaisseur mesurée sur la carcasse.

Les divers graphiques se rapportent aux différents niveaux de la couche de lard (Cou, Dos, Rein) et aux 2 positions de prises de cliché : position couchée ou position debout. 

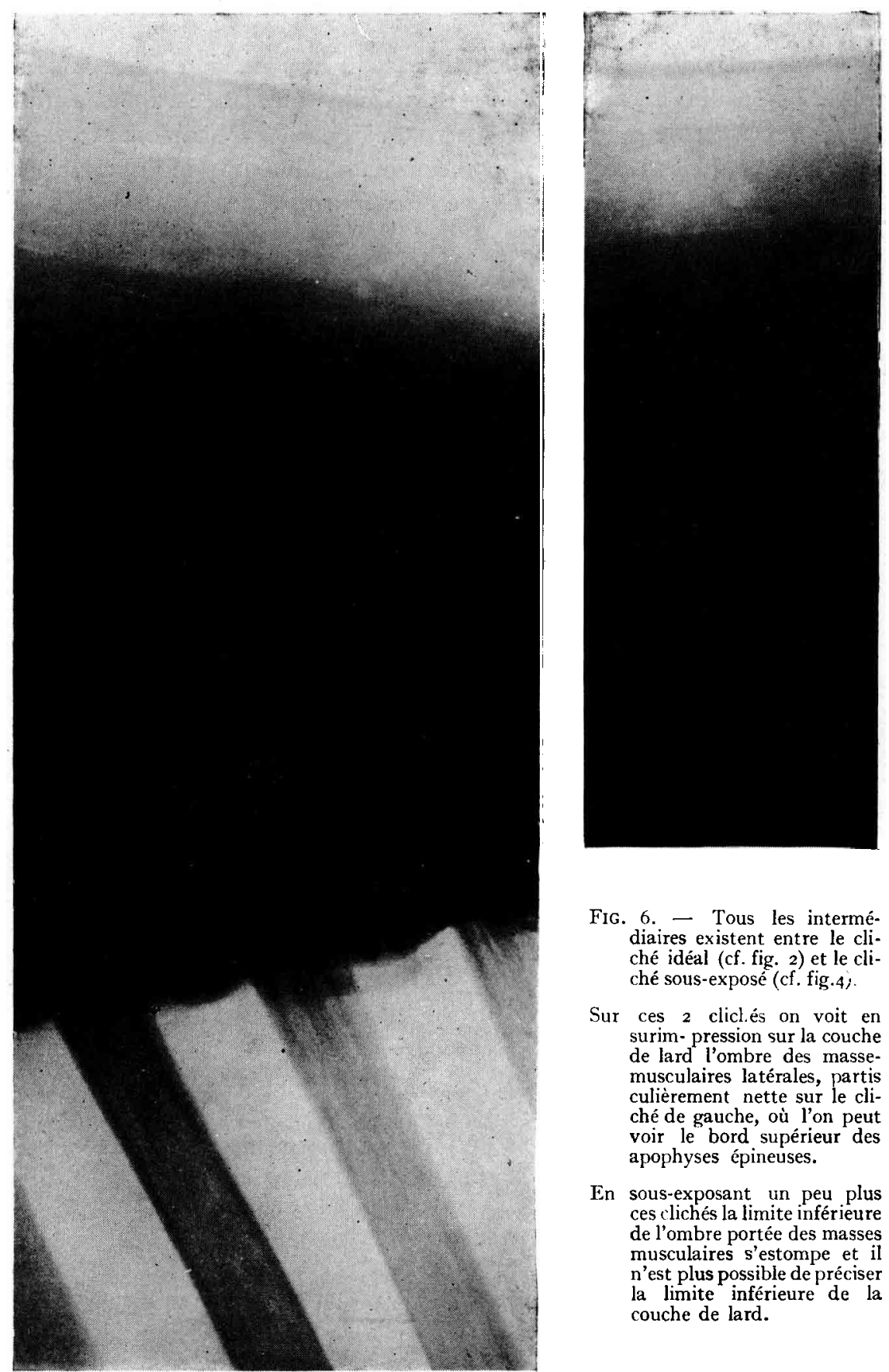

FIG. 6. - Tous les intermédiaires existent entre le cliché idéal (cf. fig. 2 ) et le cliché sous-exposé (cf. fig. 4 ;

Sur ces 2 clicl.és on voit en surim-pression sur la couche de lard l'ombre des massemusculaires latérales, partis culièrement nette sur le cliché de gauche, où l'on peut voir le bord supérieur des apophyses épineuses.

En sous-exposant un peu plus ces clichés la limite inférieure de l'ombre portée des masses musculaires s'estompe et il n'est plus possible de préciser la limite inférieure de la couche de lard. 


\section{RÉSUMÉ}

Une méthode de mesure d'épaisseur du lard in vivo a été mise au point. Elle utilise un appareil portatif de faible puissance et peut s'appliquer à des porcs de la naissance à Ioo $\mathrm{kg}$. Elle est applicable à la mesure de la couche de lard dans la région dorsale et lombaire de l'animal. Eille donne des résultats concordant avec ceux fournis après abattage des animaux.

Reçu pour publication le I7 octobre I956.

\section{RÉFERENCES BIBLIOGRAPHIQUES}

(I) Mc MeEkan (C. P.). - J. Agric. Sci., 30, 276, I940.

(2) Production et commercialisation de la viande. C. R. IIIe Journées d'Etudes F. E. Z. Sienne, I953.

(3) Leroy (A. M.) et Fúvrier (R.). - Ann. A gron., 6, I949.

(4) Charlet (P.) et F́́́vrier (R). - Ann. Nutrit. Aliment., 6, 133-58, I952.

(5) FÉvrier (R.). - C. R. A cad. A gric., 20 janvier 1954.

(6) Begon (G.), Févrięr (R.), et Zert (P.). - C.R. Acad. A gric., 20 octobre I955.

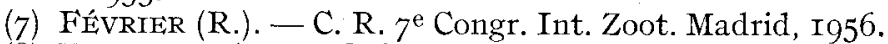

(8) Nyi,in. - Acta med. Scandinav., 31, I-207, I929.

(9) KeYs (A.), BROZEK (J.). - Physiol. Rev., 33, 245-325, 1953.

(IO) HOGREvE. - Zeitschrift f. Züchtung., XI, 377-96, I938.

(II) Veterinary Record, 29 septembre I95I.

(I2) Veterinary Record, 30 août I952. 